\title{
Recurrent leiomyoma of the oesophagus
}

\author{
RJ STANDERFER, M PANETH \\ From the Brompton Hospital, London
}

Over 850 cases of oesophageal leiomyoma have been reported worldwide and a recent survey indicates that they comprise two-thirds of all benign tumours found there. ${ }^{1}$ The usual presentation is a solitary mass of variable size, with only 28 instances of multiple simultaneous tumours. ${ }^{1-5}$ Resection is usually complete, with only one possible reported recurrence-in a patient who had had an oesophageal tumour of unknown type removed four years before surgery for a leiomyoma. ${ }^{5}$ This paper presents the first documented case of recurrent leiomyoma of the oesophagus, occurring 15 years after the original removal.

\section{Case report}

In June 1962 an otherwise healthy 51-year-old man developed severe left-sided pleuritic chest pain. Chest radiography showed a large lobulated opacity in the left lower hemithorax, pushing the left bronchus posteriorly. A barium swallow showed the distal oesophagus to be deviated to the right by extrinsic compression. Tomograms confirmed a mass, which did not contain calcium. Physical examination at the Brompton Hospital showed no abnormality. Bronchoscopy showed compression of the left main and lower-lobe bronchi. Through a left lateral thoracotomy an encapsulated tumour arising from the muscular layers of the oesophagus was exposed. It was fatty and lobulated, enveloping the oesophagus at its upper limit and centred on a large anterior tongue extending behind the heart into the mediastinum. Care was taken to enucleate the tumour completely because of its adherence to the pericardium and left inferior pulmonary vein. A second, smaller nodule was removed from the inferior pulmonary ligament. These masses measured $20 \times 20 \times$ $10 \mathrm{~cm}$ and $4 \times 2 \times 2 \mathrm{~cm}$ and histologically were leiomyomas undergoing fibrosis. The larger mass had an area of infarction, which was probably responsible for the initial pain. The patient's recovery was uneventful.

In February 1977 he presented again after routine chest radiography had showed a large right lower chest mass (fig 1). A barium swallow showed deviation of the oesophagus to the right, concentric narrowing of the distal $15 \mathrm{~cm}$, and proximal dilatation (fig 2); these appearances were again consistent with external compression. An operation (delayed because of a car accident) was performed in February 1978 . Through a right posterolateral thoracotomy a fibrous and fatty tumour was found to fill the posterior half of the hemithorax from the diaphragm to above the azygos arch. A separate chain of nodules, $2 \mathrm{~cm}$ in diameter, was

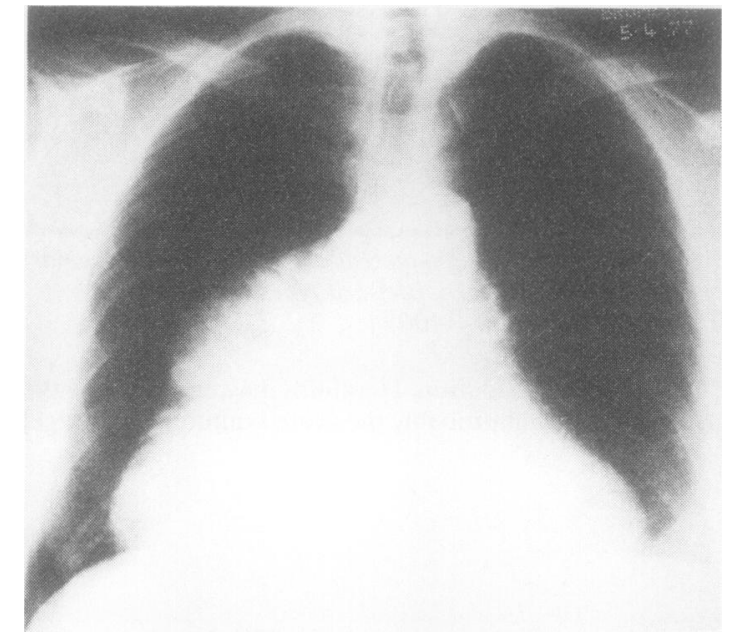

Fig 1 Chest radiograph showing a large mass in the right lower chest medially.

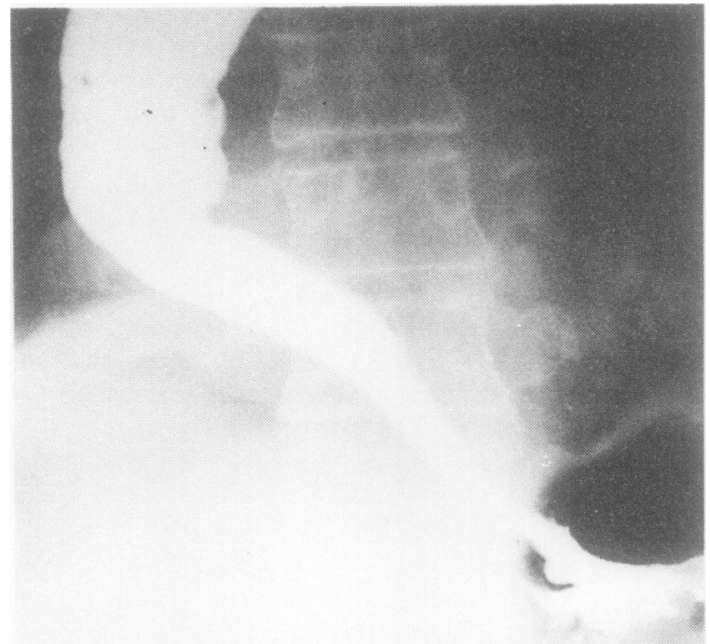

Fig 2 Barium swallow showing the oesophagus displaced to the right and compressed in its lower third. 
present near the hilum of the right lung. The encapsulated tumour was densely adherent to the muscular layers of the distal oesophagus; measured $17 \times 3 \times 5 \mathrm{~cm}$; and histologically was a leiomyoma composed of smooth muscle fibres, adipose tissue, and areas of myxomatous degeneration.

Postoperatively there was continuous drainage of chyle from the chest tubes, necessitating a second operation two weeks later. Five small chylous fistulas along the plane of oesophageal dissection were identified and ligated. Recovery was rapid and complete. The patient has remained symptom free.

\section{Discussion}

Over $90 \%$ of oesophageal leiomyomas occur from 20 to 60 years of age with a male:female ratio of $1 \cdot 9: 1$. They are one-fiftieth as common as oesophageal carcinomas and have a peak occurrence 20 years earlier. At least half are asymptomatic, one being found in every 1119 necropsies. ${ }^{1}$ In size they range up to $5 \mathrm{~kg}$. When symptoms are present dysphagia, chest pain, and pyrosis occur with equal frequency. With oesophageal obstruction weight loss related to poor nutrition or aspiration on swallowing may occur; $97 \%$ are intramural, $1 \%$ intraluminal polyps, and $2 \%$ extramural. Some $10 \%$ are situated round the circumference of the oesophagus and $2 \%$ are in the form of multiple nodules. ${ }^{15}$

On chest radiographs they appear as smooth, round, or lobulated mediastinal densities. Barium swallow usually shows a characteristic picture of a smoothly rounded defect impinging on the lumen. There is often proximal dilatation and a sharp shelf where the tumour begins. The mucosa is smooth with folds flattened out, and when seen "en face" the barium column may split on either side of the mass. Barrett ${ }^{2}$ pointed out that the radiographic appearance is not always accurate-for instance, when enlarged mediastinal glandular metastases impinge on the oesophagus and obscure an area of distal carcinoma. Similarly, circumferential tumours may produce a tortuous stricture similar to that produced by a carcinoma. ${ }^{2}$ Oesophagoscopy is therefore necessary, and usually shows an intact mucosa with extrinsic compression of the lumen. Biopsy through normal mucosa is not recommended because of the danger of perforation.

Definitive treatment is usually by transpleural enucleation, which can be accomplished in $90 \%$ of cases. Large tumours and those densely adherent to the oesophagus or situated near the gastro-oesophageal junction may require segmental resection and anastomosis. Enucleation is quite safe: no deaths have been reported with this procedure in the last 15 years.' Patients without symptoms but with characteristic radiographic and endoscopic findings have been followed for up to 15 years without showing any change, indicating that not all tumours need to be resected. ${ }^{-}$The risk of neoplastic degeneration is very low as only one reported leiomyoma had a focus of sarcoma within its substance.

Two theories about the origin of these tumours have been advanced. The first is that they arise from pre-existing smooth muscle in the muscularis mucosa, vascular smooth muscle, or circular muscular layer. This is supported by the fact that leiomyoma is more common in the lower part of the oesophagus, where the amount of smooth muscle increases: $11 \%$ are found in the upper third, $33 \%$ in the middle third and $56 \%$ in the lower third.' The second theory is that the tumour is a hamartoma arising from hyperplasia of aberrant embryonal muscle tissue. This would explain multiple simultaneous oesophageal tumours as well as the recent finding of a girl who had leiomyomas of the oesophagus, uterus, and vulva. ${ }^{4}$

Our patient undoubtedly had multicentric leiomyomas, as indicated by the two separate tumours found at the first operation and the hilar string of nodules found at the second. The large tumour found at the second operation could have been a recurrence of incompletely resected tumour or growth of a missed separate nodule. Clearly leiomyoma of the oesophagus is a slow-growing tumour and missed nodules or recurrences may be successfully dealt with by later operations.

\section{References}

'Seremitis MG, Debuzman VC, Lyons WS, Peabody JW. Leiomyoma of the oesophagus: a report of 19 surgical cases. Ann Thorac Surg 1973;16:308-16.

${ }^{2}$ Barrett NR. Benign smooth muscle tumours of the oesophagus. Thorax 1964;19:185.

${ }^{3}$ Godard JE, McCrane D. Multiple leiomyomas of the oesophagus. Am J Roentgenol 1973;117:259-62.

4 Shaffer H. Multiple leiomyomas of the oesophagus. Radiology 1976;118:29-34.

5 Storey CF, Adams WC. Leiomyoma of the oesophagus: report of 4 cases and review of the surgical literature. Am J Surg 1956;91:3-23.

- Glanz I, Greenbaum M. The radiological approach to leiomyoma of the oesophagus with long-term follow-up. Clin Radiol 1977;28:197-200. 